\title{
First order nonhomogeneous linear differential polynomials
}

\author{
Benharrat Belaidi
}




\title{
FIRST ORDER NONHOMOGENEOUS LINEAR DIFFERENTIAL POLYNOMIALS
}

\author{
BENHARRAT BELAÏDI
}

Received 10 June, 2009

\begin{abstract}
In this paper, we investigate the complex oscillation of the nonhomogeneous linear differential polynomial $g_{f}=d_{1} f^{\prime}+d_{0} f+b$. Here $d_{0}(z), d_{1}(z), b(z)$ are meromorphic functions such that at least one of $d_{0}(z)$ and $d_{1}(z)$ does not vanish identically with $\rho_{p}\left(d_{j}\right)<\infty$ $(j=0,1), \rho_{p}(b)<\infty$, and $f$ is a solution of the differential equation $f^{\prime \prime}+A(z) f=0$, where $A(z)$ is a transcendental meromorphic function with finite iterated order $\rho_{p}(A)=\rho>0$.

2000 Mathematics Subject Classification: 34M10, 30D35

Keywords: differential polynomials, linear differential equations, iterated order, iterated exponent of convergence of the sequence of distinct zeros, iterated exponent of convergence of the sequence of distinct fixed points
\end{abstract}

\section{INTRODUCTION AND MAIN RESULT}

For the definition of the iterated order of a meromorphic function, we use the same definition as in [3]([2], p. 317), ([4], p. 129). For all $r \in \mathbb{R}$, we define $\exp _{1} r:=e^{r}$ and $\exp _{p+1} r:=\exp \left(\exp _{p} r\right), p \in \mathbb{N}$. We also define for all sufficiently large $r$ the functions $\log _{1} r:=\log r$ and $\log _{p+1} r:=\log \left(\log _{p} r\right), p \in \mathbb{N}$. Moreover, we denote by $\exp _{0} r:=r, \log _{0} r:=r, \log _{-1} r:=\exp _{1} r$ and $\exp _{-1} r:=\log _{1} r$.

Definition $1([3,4])$. Let $f$ be a meromorphic function. Then the iterated $p-$ order $\rho_{p}(f)$ of $f$ is defined by

$$
\rho_{p}(f)=\varlimsup_{r \rightarrow+\infty} \frac{\log _{p} T(r, f)}{\log r} \quad(p \geq 1 \text { is an integer })
$$

where $T(r, f)$ is the Nevanlinna characteristic function of $f$. For $p=1$, this notation is called order and for $p=2$ hyper-order (see [7]). 
Definition 2 ([3]). The finiteness degree of the order of a meromorphic function $f$ is defined by

$$
i(f)=\left\{\begin{array}{cl}
0, & \text { if } f \text { is rational, } \\
\min \left\{j \in \mathbb{N}: \rho_{j}(f)<+\infty\right\}, & \text { if } f \text { is transcendental such that } \\
& \text { some } j \in \mathbb{N} \text { with } \rho_{j}(f)<+\infty \text { exists, } \\
+\infty, & \text { for } f \text { with } \rho_{j}(f)=+\infty \text { for all } j \in \mathbb{N} .
\end{array}\right.
$$

Definition 3 ([3,5]). Let $f$ be a meromorphic function. Then the iterated exponent of convergence of the sequence of distinct zeros of $f(z)$ is defined by

$$
\bar{\lambda}_{p}(f)=\varlimsup_{r \rightarrow+\infty} \frac{\log _{p} \bar{N}\left(r, \frac{1}{f}\right)}{\log r} \quad(p \geq 1 \text { is an integer }),
$$

where $\bar{N}\left(r, \frac{1}{f}\right)$ is the counting function of distinct zeros of $f(z)$ in $\{z:|z|<r\}$. For $p=1$, this notation is called the exponent of convergence of the sequence of distinct zeros and for $p=2$ the hyper-exponent of convergence of the sequence of distinct zeros (see [6]).

Definition 4 ([5]). Let $f$ be a meromorphic function. Then the iterated exponent of convergence of the sequence of distinct fixed points of $f(z)$ is defined by

$$
\bar{\tau}_{p}(f)=\bar{\lambda}_{p}(f-z)=\varlimsup_{r \rightarrow+\infty} \frac{\log _{p} \bar{N}\left(r, \frac{1}{f-z}\right)}{\log r} \quad(p \geq 1 \text { is an integer }) .
$$

For $p=1$, this notation is called the exponent of convergence of the sequence of distinct fixed points and for $p=2$ the hyper-exponent of convergence of the sequence of distinct fixed points (see [6]). Thus $\bar{\tau}_{p}(f)=\bar{\lambda}_{p}(f-z)$ is an indication of oscillation of distinct fixed points of $f(z)$.

Let $\mathscr{L}(\mathbf{G})$ denote a differential subfield of the field $\mathcal{M}(\mathbf{G})$ of meromorphic functions in a domain $\mathbf{G} \subset \mathbb{C}$. If $\mathbf{G}=\mathbb{C}$, we simply use $\mathscr{L}$ instead of $\mathscr{L}(\mathbb{C})$. A special case of such differential subfields is

$$
\mathscr{L}_{p+1, \rho}=\left\{g \text { meromorphic: } \rho_{p+1}(g)<\rho\right\},
$$

where $\rho$ is a positive constant.

Consider the linear differential equation

$$
f^{\prime \prime}+A(z) f=0,
$$

where $A(z)$ is a transcendental meromorphic function with finite iterated order $\rho_{p}(A)=\rho>0$. Since the beginning of the last four decades, a substantial number of research articles have been written to describe the fixed points of general transcendental meromorphic functions (see [8]). However, there are few studies on the fixed points of solutions of general differential equations. In [5] Laine and Rieppo have 
investigated the fixed points and iterated order of equation (1.5) and have obtained the following result:

Theorem 1 ([5]). Let $A(z)$ be a transcendental meromorphic function of finite iterated order $\rho_{p}(A)=\rho>0$ such that $\delta(\infty, A)=\lim _{r \rightarrow+\infty} \frac{m(r, A)}{T(r, A)}=\delta>0$, and let $f$ be a transcendental meromorphic solution of equation (1.5). Suppose, moreover, that either:

(i) all poles of $f$ are of uniformly bounded multiplicity or

(ii) $\delta(\infty, f)>0$.

Then $\rho_{p+1}(f)=\rho_{p}(A)=\rho$. Moreover, let

$$
P[f]=P\left(f, f^{\prime}, \ldots, f^{(m)}\right)=\sum_{j=0}^{m} p_{j} f^{(j)}
$$

be a linear differential polynomial with coefficients $p_{j} \in \mathscr{L}_{p+1, \rho}$, assuming that at least one of the coefficients $p_{j}$ does not vanish identically. Then for the fixed points of $P[f]$, we have $\bar{\tau}_{p+1}(P[f])=\rho$, provided that neither $P[f]$ nor $P[f]-z$ vanishes identically.

Remark 1 ([5], p. 904). In Theorem 1, in order to study $P[f]$, the authors consider $m \leq 1$. Indeed, if $m \geq 2$, we obtain, by repeated differentiation of (1.5), that $f^{(k)}=$ $q_{k, 0} f+q_{k, 1} f^{\prime}, q_{k, 0}, q_{k, 1} \in \mathscr{L}_{p+1, \rho}$ for $k=2, \ldots, m$. Substitution into (1.6) yields the required reduction.

The main purpose of this paper is to investigate the fixed points of the nonhomogeneous linear differential polynomial $g_{f}=d_{1} f^{\prime}+d_{0} f+b$, where $d_{0}(z), d_{1}(z)$, $b(z)$ are meromorphic functions generated by solutions of equation (1.5). Instead of looking at the zeros of $g_{f}-z$, we proceed to a slight generalization by considering zeros of $g_{f}-\varphi(z)$, where $\varphi$ is a meromorphic function of finite iterated $p$-order, while the solution of respective differential equation is of infinite iterated $p-$ order.

Let us denote by

$$
\begin{gathered}
\alpha_{0}=d_{0}^{\prime}-d_{1} A, \quad \alpha_{1}=d_{0}+d_{1}^{\prime}, \\
h=d_{1} \alpha_{0}-d_{0} \alpha_{1},
\end{gathered}
$$

where $A, d_{j}(j=0,1)$ are meromorphic functions. We obtain:

Theorem 2. Let $A(z)$ be a transcendental meromorphic function of finite iterated order $\rho_{p}(A)=\rho>0$ such that $\delta(\infty, A)=\delta>0$, let $d_{0}(z), d_{1}(z), b(z)$ be meromorphic functions such that at least one of $d_{0}(z), d_{1}(z)$ does not vanish identically with $\rho_{p}\left(d_{j}\right)<\infty(j=0,1), \rho_{p}(b)<\infty$ such that $h \neq \equiv$. Let $\varphi(z)(\not \equiv 0)$ be a meromorphic function of finite iterated $p-$ order such that $d_{1}\left(\varphi^{\prime}-b^{\prime}\right)-\alpha_{1}(\varphi-b) \not \equiv 0$. Suppose, moreover, that either: 
(i) all poles of $f$ are of uniformly bounded multiplicity or

(ii) $\delta(\infty, f)>0$.

If $f(z) \not \equiv 0$ is a meromorphic solution of (1.5), then the differential polynomial $g_{f}=d_{1} f^{\prime}+d_{0} f+b$ satisfies $\bar{\lambda}_{p}\left(g_{f}-\varphi\right)=\rho_{p}(f)=+\infty$ and $\bar{\lambda}_{p+1}\left(g_{f}-\varphi\right)=$ $\rho_{p+1}(f)=\rho_{p}(A)=\rho$.

Setting $p=1$ and $\varphi(z)=z$ in Theorem 2, we obtain the following corollary:

Corollary 1. Let $A(z)$ be a transcendental meromorphic function of finite order $\rho(A)=\rho>0$ such that $\delta(\infty, A)=\delta>0$, let $d_{0}(z), d_{1}(z), b(z)$ be meromorphic functions such that at least one of $d_{0}(z), d_{1}(z)$ does not vanish identically with $\rho\left(d_{j}\right)<\infty(j=0,1), \rho(b)<\infty$ such that $h \not \equiv 0$ and $d_{1}\left(1-b^{\prime}\right)-\alpha_{1}(z-b) \not \equiv$ 0 . Suppose, moreover, that either:

(i) all poles of $f$ are of uniformly bounded multiplicity or

(ii) $\delta(\infty, f)>0$.

If $f(z) \not \equiv 0$ is a meromorphic solution of (1.5), then the differential polynomial $g_{f}=$ $d_{1} f^{\prime}+d_{0} f+b$ has infinitely many fixed points and satisfies $\bar{\tau}\left(g_{f}\right)=\rho(f)=+\infty$, $\bar{\tau}_{2}\left(g_{f}\right)=\rho_{2}(f)=\rho(A)=\rho$.

\section{Some LemMas}

We need the following lemmas in the proofs of our theorem.

Lemma 1 (see Remark 1.3 of [3]). If $f$ is a meromorphic function with $i(f)=$ $p \geq 1$, then $\rho_{p}(f)=\rho_{p}\left(f^{\prime}\right)$.

Lemma 2 ([5]). If $f$ is a meromorphic function with $0<\rho_{p}(f)<\rho(p \geq 1)$, then $\rho_{p+1}(f)=0$.

Lemma 3 ([1]). Let $k \geq 2$ and $A(z)$ be a transcendental meromorphic function of finite iterated order $\rho_{p}(A)=\rho>0$ such that $\delta(\infty, A)=\delta>0$. Suppose, moreover, that either:

(i) all poles of $f$ are of uniformly bounded multiplicity or

(ii) $\delta(\infty, f)>0$.

Then every meromorphic solution $f(z) \not \equiv 0$ of

$$
f^{(k)}+A(z) f=0
$$

satisfies $i(f)=p+1, \rho_{p}(f)=+\infty$ and $\rho_{p+1}(f)=\rho_{p}(A)=\rho$.

Lemma 4 ([1]). Let $A_{0}, A_{1}, \ldots, A_{k-1}, F \not \equiv 0$ be finite iterated $p$-order meromorphic functions. If $f$ is a meromorphic solution with $\rho_{p}(f)=+\infty$ and $\rho_{p+1}(f)=$ $\rho<+\infty$ of the equation

$$
f^{(k)}+A_{k-1} f^{(k-1)}+\cdots+A_{1} f^{\prime}+A_{0} f=F,
$$


then $\bar{\lambda}_{p}(f)=\rho_{p}(f)=+\infty$ and $\bar{\lambda}_{p+1}(f)=\rho_{p+1}(f)=\rho$.

Lemma 5. Let $A(z)$ be a transcendental meromorphic function with finite iterated order $\rho_{p}(A)=\rho>0$ such that $\delta(\infty, A)=\delta>0$. Let $d_{0}(z), d_{1}(z), b(z)$ be meromorphic functions such that at least one of $d_{0}(z), d_{1}(z)$ does not vanish identically with $\rho_{p}\left(d_{j}\right)<\infty(j=0,1), \rho_{p}(b)<\infty$ such that $h \neq \equiv 0$. Suppose, moreover, that either:

(i) all poles of $f$ are of uniformly bounded multiplicity or

(ii) $\delta(\infty, f)>0$.

If $f(z) \not \equiv 0$ is a meromorphic solution of (1.5), then the differential polynomial

$$
g_{f}=d_{1} f^{\prime}+d_{0} f+b
$$

satisfies $i\left(g_{f}\right)=p+1, \rho_{p}\left(g_{f}\right)=\rho_{p}(f)=+\infty$ and $\rho_{p+1}\left(g_{f}\right)=\rho_{p+1}(f)=$ $\rho_{p}(A)=\rho$.

Proof. Suppose that $f(\not \equiv 0)$ is a meromorphic solution of equation (1.5). Then by Lemma 3, we have $\rho_{p}(f)=+\infty$ and $\rho_{p+1}(f)=\rho_{p}(A)=\rho$. Differentiating both sides of equation (2.3) and replacing $f^{\prime \prime}$ with $f^{\prime \prime}=-A f$, we obtain

$$
g_{f}^{\prime}-b^{\prime}=\left(d_{0}+d_{1}^{\prime}\right) f^{\prime}+\left(d_{0}^{\prime}-d_{1} A\right) f \text {. }
$$

Then by (1.7), (2.3) and (2.4), we have

$$
\begin{aligned}
& d_{1} f^{\prime}+d_{0} f=g_{f}-b, \\
& \alpha_{1} f^{\prime}+\alpha_{0} f=g_{f}^{\prime}-b^{\prime} .
\end{aligned}
$$

Set

$$
h=d_{1} \alpha_{0}-\alpha_{1} d_{0}=d_{1}\left(d_{0}^{\prime}-d_{1} A\right)-d_{0}\left(d_{0}+d_{1}^{\prime}\right) .
$$

By the condition $h \not \equiv 0$ and (2.5) - (2.7), we get

$$
f=\frac{d_{1}\left(g_{f}^{\prime}-b^{\prime}\right)-\alpha_{1}\left(g_{f}-b\right)}{h} .
$$

If $\rho_{p}\left(g_{f}\right)<+\infty$, then by (2.8) and Lemma 1 we obtain $\rho_{p}(f)<+\infty$, and this is a contradiction. Hence $\rho_{p}\left(g_{f}\right)=\rho_{p}(f)=+\infty$.

Now, we prove that $\rho_{p+1}\left(g_{f}\right)=\rho_{p+1}(f)=\rho$. By (2.3), Lemma 1 and Lemma 2 , we get $\rho_{p+1}\left(g_{f}\right) \leq \rho_{p+1}(f)$ and by $(2.8)$ we have $\rho_{p+1}(f) \leq \rho_{p+1}\left(g_{f}\right)$. This yield $\rho_{p+1}\left(g_{f}\right)=\rho_{p+1}(f)=\rho$.

Remark 2. In Lemma 5 , if we don't have the condition $h \not \equiv 0$, then the differential polynomial can be of finite iterated $p$-order. For example, if $d_{0}^{\prime}-d_{1} A \equiv 0$ and $d_{1}^{\prime}+$ $d_{0} \equiv 0$, then $h \equiv 0$ and $g_{f}^{\prime}-b^{\prime} \equiv 0$. It follows that $\rho_{p}\left(g_{f}\right)=\rho_{p}\left(g_{f}^{\prime}\right)=\rho_{p}\left(b^{\prime}\right)=$ $\rho_{p}(b)<+\infty$. Hence, the condition $h \not \equiv 0$ is necessary in Theorem 2 . 


\section{Proof of Theorem 2}

Suppose that $f(z) \not \equiv 0$ is a meromorphic solution of (1.5). Then by Lemma 3, we have $\rho_{p}(f)=+\infty$ and $\rho_{p+1}(f)=\rho_{p}(A)=\rho$. By Lemma 5, it is clear that $g_{f} \not \equiv 0$ and $g_{f} \not \equiv \varphi$. Set $w(z)=d_{1} f^{\prime}+d_{0} f+b-\varphi$, since $\rho_{p}(\varphi)<+\infty$, then by Lemma 5 we have $\rho_{p}(w)=\rho_{p}\left(g_{f}\right)=\rho_{p}(f)=+\infty$ and $\rho_{p+1}(w)=\rho_{p+1}\left(g_{f}\right)=$ $\rho_{p+1}(f)=\rho_{p}(A)=\rho$. In order to prove $\bar{\lambda}_{p}\left(g_{f}-\varphi\right)=+\infty$ and $\bar{\lambda}_{p+1}\left(g_{f}-\varphi\right)=$ $\rho_{p}(A)=\rho$, we need to prove only $\bar{\lambda}_{p}(w)=+\infty$ and $\bar{\lambda}_{p+1}(w)=\rho_{p}(A)=\rho$. Substituting $g_{f}=w+\varphi$ into (2.8)

$$
f=\frac{d_{1} w^{\prime}-\alpha_{1} w}{h}+\psi
$$

where

$$
\psi=\frac{d_{1}\left(\varphi^{\prime}-b^{\prime}\right)-\alpha_{1}(\varphi-b)}{h} .
$$

Substituting (3.1) into equation (1.5), we obtain

$$
\frac{d_{1}}{h} w^{\prime \prime \prime}+\phi_{2} w^{\prime \prime}+\phi_{1} w^{\prime}+\phi_{0} w=-\left(\psi^{\prime \prime}+A(z) \psi\right)=W,
$$

where $\phi_{j}(j=0,1,2)$ are meromorphic functions with $\rho_{p}\left(\phi_{j}\right)<\infty(j=0,1,2)$. By $\rho_{p}(\psi)<+\infty$ and the condition $\psi \neq \equiv 0$, it follows by Lemma 3 that $W \neq \equiv 0$. By Lemma 4 , we obtain $\bar{\lambda}_{p}(w)=\rho_{p}(w)=+\infty$ and $\bar{\lambda}_{p+1}(w)=\rho_{p+1}(w)=\rho$, i.e., $\bar{\lambda}_{p}\left(g_{f}-\varphi\right)=\rho_{p}(f)=+\infty$ and $\bar{\lambda}_{p+1}\left(g_{f}-\varphi\right)=\rho_{p+1}(f)=\rho_{p}(A)=\rho$.

Remark 3. From the proof of Theorem 2, we see that the condition $d_{1}\left(\varphi^{\prime}-b^{\prime}\right)-$ $\alpha_{1}(\varphi-b) \not \equiv 0$ is necessary because if $d_{1}\left(\varphi^{\prime}-b^{\prime}\right)-\alpha_{1}(\varphi-b) \equiv 0$, then $\psi \equiv 0$ and $W \equiv 0$.

\section{REFERENCES}

[1] B. Belaïdi, "Oscillation of fixed points of solutions of some linear differential equations," Acta Math. Univ. Comenian. (N.S.), vol. 77, no. 2, pp. 263-269, 2008.

[2] L. G. Bernal, "On growth $k$-order of solutions of a complex homogeneous linear differential equation,” Proc. Amer. Math. Soc., vol. 101, no. 2, pp. 317-322, 1987.

[3] L. Kinnunen, "Linear differential equations with solutions of finite iterated order," Southeast Asian Bull. Math., vol. 22, no. 4, pp. 385-405, 1998.

[4] I. Laine, Nevanlinna theory and complex differential equations, ser. de Gruyter Studies in Mathematics. Berlin: Walter de Gruyter \& Co., 1993, vol. 15.

[5] I. Laine and J. Rieppo, "Differential polynomials generated by linear differential equations," Complex Var. Theory Appl., vol. 49, no. 12, pp. 897-911, 2004.

[6] M.-S. Liu and X.-M. Zhang, "Fixed points of meromorphic solutions of higher order linear differential equations," Ann. Acad. Sci. Fenn. Math., vol. 31, no. 1, pp. 191-211, 2006.

[7] C.-C. Yang and H.-X. Yi, Uniqueness theory of meromorphic functions, ser. Mathematics and its Applications. Dordrecht: Kluwer Academic Publishers Group, 2003, vol. 557. 
[8] Q. T. Zhang and C. C. Yang, The Fixed Points and Resolution Theory of Meromorphic Functions. Beijing: Beijing University Press, 1988.

\section{Author's address}

Benharrat Belaïdi

University of Mostaganem, Department of Mathematics, Laboratory of Pure and Applied Mathematics, B. P. 227 Mostaganem, Algeria

E-mail address: belaidi@univ-mosta.dz 